\title{
Klaus Pietschmann
}

\section{Einführung}

Nachdem sich troja 2013 mit Polen, Schlesien und Böhmen befasst hat, erfolgte 2015 der Sprung über den Atlantik. Der Spurensuche an den östlichen Rändern eines Kulturraums, der im 15. und 16. Jahrhundert auch durch die frankoflämische Vokalpolyphonie in ihren unterschiedlichen Ausprägungen gekennzeichnet war, stand nunmehr der Blick auf die »Neue Welt« gegenüber, deren Eroberung und ideelle Eingliederung in die frühneuzeitliche europäische Staatenwelt mit einem Export der grundsätzlich selben Vokalpolyphonie einherging. Grenzgebiete in beiden Fällen, in denen musikstilistische Standards europäischer Eliten auf regionale Traditionen prallten und mit diesen in vielfältiger Weise interagierten.

Dieser Vergleich ist zweifellos grob vereinfachend, aber er mag verdeutlichen, dass die Beschäftigung mit »Vokalpolyphonie zwischen Alter und Neuer Welt« im Rahmen eines Symposiums zur Renaissancemusikforschung weit weniger exotisch ist, als es auf Anhieb erscheinen mag. Mit der Errichtung der ersten Bistümer in Lateinamerika wurde neben der Kirchenarchitektur des Siglo de Oro auch das Repertoire der spanischen Kathedralkirchen in die neuen Besitzungen transferiert und mittels dieser Repräsentationskultur die kulturelle Homogenität des erweiterten Imperiums postuliert. $\mathrm{Zu}$ den spektakulärsten Manifestationen dieses Postulats zählte der Tumulo Imperial de la gran cindad de Mexico, den der Vizekönig Luis de Velasco 1559 anlässlich des Todes von Karl V. errichten ließ: In seiner 1560 gedruckten Beschreibung ging Francisco Cervantes de Salazar auch ausführlich auf die Gesänge ein und betonte ausdrücklich die Aufführung mehrerer Motetten von Cristóbal de Morales: »Se dixo el Parce mihi domine, de canto de organo compuesto de Morales: que dio gran contento oyrle. ${ }^{1}$

Mit dem Transfer von Repertoires engstens verknüpft waren jedoch von Beginn an vielfältige Akkulturationsprozesse: Schon die ersten Franziskanermönche um Pedro de Gante, die 1523 im Auftrag Karls V. zur Missionierung Neuspaniens aufbrachen, setzten offenbar gleichermaßen auf die Implementierung indigener Musik in den Gottesdienst und die Unterweisung in europäischer Vokalpolyphonie, um ihre Ziele zu erreichen. Die dabei erzielten Erfolge dokumentieren die Bemühungen des ersten Bischofs von Mexico Juan de Zumárraga, der zu Beginn der 1540er Jahre den polyphonen Gesang und die Notenkopiatur der Indios lob-

1 Robert Stevenson, Music in Mexico: A Historical Survey, New York 1952, S. 89. 
te ${ }^{2}$ aber auch die Vision des ersten Heiligen der Neuen Welt Juan Diego, dem die Virgen de Guadalupe 1531 auf den Ruinen des Aztekentempels von Tepeyac ganz im Einklang mit europäischen Mirakelberichten unter süßem Duft und vielstimmigem Engelsgesang erschien. Auch wenn diese Berichte auf europäische Erwartungshaltungen gemünzt waren und ihre Aussagekraft entsprechend zu relativieren ist, so lassen etwa die großdimensionierten Guidonischen Hände an den Wänden etlicher amerikanischer Konvente keinen Zweifel daran, dass traditionellen europäischen Methoden der musikalischen Unterweisung ein zentraler Stellenwert in der Mission zukam. ${ }^{3}$

Die Rolle der Musik in der Mission und die musikalischen Wechselbeziehungen zwischen Europa und der sog. »Neuen Welt« sind schon seit langem ein Gegenstand umfänglicher Forschungen. Ausgehend von den Pionierleistungen Robert Stevensons, der seit den 1950er Jahren in seinem langen Forscherleben (er starb 2012 im Alter von 96 Jahren) zahlreiche Monographien und Studien zur Musik Lateinamerikas vorlegte, hat sich das Forschungsfeld vor allem im spanischsprachigen und anglophonen Raum kontinuierlich entwickelt und etabliert. An dieser Stelle erwähnt seien nur einige der wichtigsten Publikationen der letzten Jahre: Geoffrey Baker und Tess Knighton gaben 2011 den Band Music and Urban Society in Colonial Latin America heraus, der an die raumsoziologischen Studien zur frühneuzeitlichen Musikgeschichte der letzten Jahre anknüpft und die Rolle der Musik bei kirchlichen und politischen Festen und Ritualen in unterschiedlichen Zentren Lateinamerikas fokussiert. ${ }^{4}$ Methodisch ähnliche Wege beschritten die Kolloquien des Forschungsverbundes Musicat mehrerer mexikanischer Universitäten, die seit 2004 stattfanden und sich der Musik- und Festkultur an neuspanischen Kathedralen widmeten. ${ }^{5}$ Stärker auf die Fragestellung dieses Symposiums ausgerichtet ist der Band La Música y el Atlantico. Relaciones musicales entre España y Latinoamérica, den María Gembero Ustárroz und Emilio Ros-Fábregas 2007 herausgaben; ${ }^{6}$ die Schlaglichter, die hier auf den musikali-

2 Ders., Music in Aztec and Inca Territory, Berkeley and Los Angeles 1968, S. 162. Vgl. José Marín López, »Musical Cultures in the Reinos de Indias«, in: Companion to Music in the Age of the Catholic Monarchs at the Time of Isabel and Ferdinand, hrsg. von Tess Knighton, Leiden und Boston 2017, S. 323-363, hier: S. 352f.

3 James A. Sandos, Converting California. Indians and Franciscans in the Missions, New Haven und London, 2004, S. 134.

4 Geoffrey Baker, Tess Knighton, Music and Urban Society in Colonial Latin America, Cambridge 2011.

5 Vgl. den Überblick über die Aktivitäten und Publikationen unter: www.musicat.unam.mx.

6 María Gembero Ustárroz, Emilio Ros-Fábregas, La Música y el Atlantico. Relaciones musicales entre España y Latinoamérica, Granada 2007. 
schen Austausch »entre dos mundos« geworfen werden, verdeutlichen das große Potential, das dieser Gegenstand noch bietet.

Viele dieser Ansätze betreiben ihrem Grundverständnis nach Regionalmusikforschung, die Einzelphänomene in ihrem jeweiligen unmittelbaren zeitlichen, räumlichen und kulturellen Kontext verortet. Dies erklärt auch, warum das frühneuzeitliche Musikleben außerhalb Europas in musikgeschichtlichen Überblicksdarstellungen nach wie vor praktisch keine Berücksichtigung findet. Und wenn in der letzten 9. Auflage von Burkholders History of Western Music ${ }^{7}$ dem Thema »Spain and the New World« immerhin 5 Seiten gewidmet werden, so wird hiermit ebenfalls die vorherrschende Auffassung fortgeschrieben, dass es sich bei diesen Entwicklungen um bestenfalls periphere Ausläufer der großen Meistererzählung vom musikalischen Kunstwerk und seinen nationalen, immer aber abendländischen Ausprägungen handelt. Hinterfragt wird diese stets unausgesprochene, vielfach wohl auch unbewusste Auffassung neuerdings immer wieder, so insbesondere im Jahr 2014 in Havanna, wo eine von der Casa de las Américas und der International Musicological Society veranstaltete Tagung zu musikalischen Kanonisierungsprozessen in den Amerikas stattfand; sie behandelte Fragen der Etablierung von musikalischen Kanones unter dem Eindruck von Mission, Revolution und Nationsbildung, die im Spannungsfeld von Eigen- und Fremdidentität angesiedelt war. Die Tagung zeigte, dass die Erarbeitung historiographischer Modelle zur Beschreibung dieser komplexen Entwicklungen erhebliches Konfliktpotential birgt. Einer nach wie vor europäisch-angloamerikanisch dominierten Scientific Community begegnen zunehmend eigenständige Fachkulturen in den Ländern Lateinamerikas, die spezifische Zugänge zu musikalischem »Erbe« und der historischen Dimension gegenwärtiger Musikkultur entwickeln.

Dieses Konfliktpotential sei an einem Beispiel kurz erläutert. Im Jahre 1931 wurde dem mexikanischen Kleriker Octaviano Valdés in Cacalomacán (Toluca, Mexiko) von den Dorfbewohnern eine Musikhandschrift übergeben, die neben vier Palestrina-Messen auch zwei kurze Kompositionen in schlichtem vierstimmigem Satz enthält, denen ein Text in Náhuatl, der Sprache der Azteken, unterlegt ist. Zugeschrieben sind sie einem »Don Hernando Franco«, der zunächst mit dem ersten Kapellmeister der Kathedrale von Mexico, Hernando Franco, identifiziert wurde. Robert Stevenson, der die Handschrift um 1950 erstmals zu Gesicht bekam, stellte allerdings fest, dass der Titel »Don« im Spanischen auf adelige Herkunft hindeute und der Kapellmeister daher nicht als Autor in Frage käme. Vielmehr stellte er die nachfolgend allgemein akzeptierte These auf, es habe sich um einen hochrangigen Azteken gehandelt, dessen Taufpate besagter Hernando Franco gewesen sei und dessen Namen er, einer gängigen Praxis folgend, ange-

7 J. Peter Burkholder, Donald Jay Grout, Claude V. Palisca, A History of Western Music, New York 2014. 
nommen habe. Vermutlich sei er ein Schüler Francos gewesen, woraus sich auch seine musikalische Expertise erkläre. ${ }^{8}$ Nachfolgend standen die beiden Kompositionen und der Codex Valdés insgesamt exemplarisch für die Akkulturation europäischer Vokalpolyphonie im kolonialen Mexico, denen sich eine Reihe weiterer nachfolgend aufgefundener Kompositionen über Texte in indigenen Sprachen an die Seite stellen ließen. Für die Schlichtheit der Kompositionen und eine Reihe von Fehlern in der Kopiatur der Palestrina-Messen wurden dabei implizit die unausgereiften Kompetenzen des Azteken verantwortlich gemacht. Mit Vehemenz in Frage gestellt wurde Stevensons These allerdings durch den mexikanischen Musikforscher Elias Israel Morado Hernández auf besagtem Kongress in Havanna, zwischenzeitlich publiziert im Boletín Musica der Casa de las Americas. ${ }^{9}$ Er führte zunächst an, dass ein »Don Hernando Franco « anderweitig nicht nachweisbar und Stevensons Autorschaftsthese zweifelhaft sei; als Hauptargument gegen das implizierte Kompetenzdefizit führte er die zahlreichen korrigierten Fehler in der Handschrift an, die sehr wohl eine Sensibilität für Fehlerhaftigkeit erkennen ließen und gegen die unterstellte Inferiorität der indigenen Schreiber und Komponisten sprächen. In Konsequenz konstatiert Morado Hernández bei Stevenson und anderen Musikhistoriographen eine Haltung, die Edmundo O'Gorman 1958 als »Invención de América « bezeichnete ${ }^{10}$ - eine Erfindung Amerikas unter der Prämisse europäischer Superiorität.

Die Stichhaltigkeit dieser Argumente interessiert hier nur am Rande: Dass korrigierte und unkorrigierte Fehler in europäischen Chorbüchern ebenso häufig anzutreffen sind wie mediokre Kompositionen, ist hinlänglich bekannt, und die generell eingeschränkte Aussagekraft von Zuschreibungen in Handschriften und Drucken des 16. Jahrhunderts verdeutlicht plastisch das bekannte Diktum, Josquin habe nach seinem Tod mehr komponiert als zu Lebzeiten. Festzuhalten ist vielmehr, dass der Fall symptomatisch für das angesprochene Konfliktpotential stehen kann: Geschichtsbilder können (nicht nur) in Lateinamerika zu Projektionsflächen einer komplexen Identitätsfindung werden, die die Auseinandersetzung mit der eigenen Geschichte bzw. ihrer Konstruktion latent oder offen in hohem Maße prägt. Dieses Phänomen begleitet und beschäftigt die historische Lateinamerikaforschung bereits seit langem. Für die Musikwissenschaft sind solche Erfahrungen indes eher neu, und sie lassen erahnen, warum sich die Musikpraxis im frühneuzeitlichen Lateinamerika auch in näherer Zukunft nicht ohne

8 Stevenson, Music in Mexico (wie Anm. 1), S. $120 \mathrm{f}$.

9 Elias Morado Hernández, »El Códice Valdés (ca. 1620-1630) y la inteligencia musical Americana indiciaria «, in: Boletín Música (2014), S. 58-74.

10 Edmundo O'Gorman, La invención de América. El universalismo de la cultura de occidente, México 1958. 
weiteres in die Narrativen musikgeschichtlicher Überblicksdarstellungen einschreiben lassen wird.

Jedoch verdeutlicht das Beispiel des Codex Valdés noch in anderer Hinsicht die Komplexität des Tagungsthemas. Dass die Handschrift exemplarisch für das Phänomen »Vokalpolyphonie zwischen Alter und Neuer Welt« stehen kann, ist evident, aber ob sie einen Austauschprozess im Sinne einer stilistischen Akkulturation oder nicht doch lediglich einen bloßen Repertoire- und Stiltransfer markiert, wird sich kaum jemals eindeutig bestimmen lassen. Stevensons These, dass es sich um eine Form der Adaption europäischer Kompositionspraxis durch einen indigenen Adepten handelt, dürfte sich ebensowenig eindeutig belegen lassen wie die andere denkbare Lesart, dass es sich bei den Kompositionen um die Schöpfungen eines Missionars handelte. Unstrittig dürfte allerdings sein, dass aus der Handschrift von indigenen Musikern gesungen wurde. Inwieweit damit aber eine Überlagerung mit indigenen Musizierpraktiken einhergegangen sein könnte, ist eine offene Frage.

Das Beispiel markiert damit anschaulich die vielfältigen Verflechtungen, denen die transatlantischen Musikkulturen der Frühen Neuzeit unterlagen, und die hier versammelten Beiträge nehmen unterschiedliche Spielarten dieses Prozesses in den Blick. Der einleitende Beitrag der Historikerin Renate Pieper öffnet dabei zunächst den Blick für die oftmals leichter greifbaren, jedoch keineswegs weniger intrikaten Interdependenzen auf der Ebene der materiellen Kultur. Für die Musikkultur bieten die zahlreichen überlieferten Berichte von Missionaren zur Musikpraxis einen zentralen Ansatzpunkt, allerdings erweist sich die Auswertung der hier vermittelten Informationen als Herausforderung: Der Beitrag von Jutta Toelle nimmt sich dieses Problems an und widmet sich dabei der Frage, in welchem Maße die Idee von Mission durch Musik von Mythen und Narrativen sui generis geprägt ist. Dem vorgelagert sind die Strategien, die im Rahmen der »inneren Mission « Europas in der Frühen Neuzeit entwickelt wurden, mit denen sich der Beitrag von Daniele Filippi befasst. Die Berichte der Missionare repräsentieren zugleich die Rückwirkung musikdidaktischer Strategien nach Europa. Ein anderes vielzitiertes, aber wenig untersuchtes musikalisches Transferphänomen in diese Richtung bilden die Tänze lateinamerikanischen Ursprungs, die Eingang in den europäischen Kanon fanden. Die Ursprungsnarrative dieser höfischen Tänze in der frühen Neuzeit hat der Beitrag von Hanna Walsdorf zum Gegenstand.

Am Beispiel des Codex Valdés lässt sich noch eine andere Problematik ablesen, mit der sich die Renaissancemusikforschung seit jeher konfrontiert sieht: Wie nämlich der Codex nach Cacalomacán gelangt war, ist keineswegs klar. Plausibel erscheint die Vermutung, dass ein Kleriker im Zuge der Revolution zu Beginn des 20. Jahrhunderts aus Angst vor Plünderungen die Handschrift zusammen mit anderen Kunstgegenständen aus einer nicht mehr lokalisierbaren Kirche den Dorfbewohnern zur Aufbewahrung übergab - ein auch anderweitig häufig be- 
zeugter Vorgang. Provenienzforschung ist in diesem Fall nicht möglich, so dass Aussagen über den Überlieferungskontext kaum zu treffen sind. Damit ist die Frage der Quellenüberlieferung berührt, die für das Symposiumsthema ebenfalls von zentraler Wichtigkeit ist. Speziell in Mexiko wurden im vergangenen Jahrzehnt wesentliche Fortschritte bei der Erschließung musikalischer Quellen erzielt - an den Kathedralkirchen von Oaxaca und Puebla sowieso teilweise auch in kleineren Dörfern wurden umfangreiche Erschließungskampagnen durchgeführt. Am weitesten fortgeschritten ist die Arbeit in Mexico City, wo die musikalischen Kathedralbestände der Kolonialzeit in einem Katalog von Javier Marín-López sowie zusätzlich in einer Online-Datenbank entsprechend den RISM-Kriterien erfasst wurden. ${ }^{11}$ Auch in anderen Ländern wie Bolivien wurden umfangreiche Quellenbestände erschlossen, hier insbesondere die Musik der reducciones jesuitas von Chiquitos und Moxos. Dem gegenüber stehen jedoch noch erhebliche nicht oder nur unvollständig erschlossene Bestände. Auch wenn sich wissenschaftsfördernde Einrichtungen Südamerikas zur Musik der Kolonialzeit zunehmend als Teil eines kulturellen Erbes bekennen, das es aufzuarbeiten gilt, sind die Ressourcen knapp und Quellenbestände mitunter schwer erreichbar, etwa wenn sie sich im kirchlichen Besitz befinden - so scheiterte der Versuch, die Chorbücher des 16. Jahrhunderts in der Kathedrale von Guatemala City in RISM zu erfassen, an den Widerständen der lokalen kirchlichen Instanzen. Umso erfreulicher ist es, dass Omar Morales Abril, der als einziger Wissenschaftler in der jüngeren Vergangenheit Zugang zu diesen reichen Beständen hatte, in seinem Beitrag einen Überblick bietet und eine Bewertung unternimmt. Noch häufiger aber sieht man sich mit der Problematik sehr weitgehender Quellenverluste für die hier interessierende Periode konfrontiert - so etwa in Brasilien, und Christian Storch befasst sich mit den Möglichkeiten, dieser methodischen Herausforderung zu begegnen.

Das Symposium wurde durch ein Gesprächskonzert beschlossen, das Guy Bovet als einer der besten Kenner historischer Orgeln in Lateinamerika an der spanischen Barockorgel der Hochschule für Musik gab; leider kann es nicht im Rahmen dieser Publikation dokumentiert werden. Gedankt sei abschließend der Johannes Gutenberg-Universität Mainz für die großzügige Finanzierung des Symposium sowie N. K. E. Hasse und Herrn Stephan Münch für die redaktionelle Betreuung und graphische Einrichtung dieser Publikation.

11 Javier Marín López, Los libros de polifonía de la Catedral de México: estudio y catálogo critico, Jaén 2012. Vgl. ferner Anm. 5. 